\title{
THE CONSTRUCTION OF GLOBAL ATTRACTORS
}

\author{
MARCY BARGE AND JOE MARTIN
}

(Communicated by Dennis Burke)

\begin{abstract}
The purpose of this note is to show that every inverse limit space of an interval mapping can be realized as a global attractor for a homeomorphism of the plane.
\end{abstract}

The purpose of this note is to describe a simple method for the construction of an abundance of global attractors. In order to facilitate understanding, we describe this method in the plane.

Suppose that $I$ is an interval and that $f: I \rightarrow I$ is continuous. Let $(I, f)$ be the inverse limit space $\left\{\left(x_{0}, x_{1}, \ldots\right) \mid x_{i} \in I\right.$ and $\left.f\left(x_{i+1}\right)=x_{i}\right\}$, with metric $d\left(\left(x_{0}, x_{1}, \ldots\right),\left(y_{0}, y_{1}, \ldots\right)\right)=\sum_{i=0}^{\infty}\left|x_{i}-y_{i}\right| / 2^{i}$. We will show that $(I, f)$ can be topologically realized as a global attractor in the plane. These inverse limit spaces are examples of what Bing has called "snakelike continua", see [Bi] or [Wa]. Furthermore, the dynamics on $(I, f)$ can be understood in terms of the dynamics of $f$, see [B-M1; B-M2; B-M3]. In [Wi], Williams discusses inverse limits of branched 1-manifolds as "generalized solenoids", which have attracting neighborhoods.

The idea is a simple one. Imagine that $D$ is a disk and that $I \subset$ int $D$. We construct a map $H: D \rightarrow D$ such that (1) $H(I)=I$, and $H \mid I=f ;$ (2) $H \mid \operatorname{Bdry}(D)=\mathrm{id}$; (3) if $x \in \operatorname{int} D$ there is a positive integer $n$, such that $H^{n}(x) \in I$; and (4) $H$ is uniformly approximated by homeomorphisms. Then let $X$ be the inverse limit of $D$ with bonding map $H$. Using (4), it follows from a theorem of $\mathrm{M}$. Brown [Br] that $X$ is a topological disk. Using conditions (1) and (3) we see that $(I, f)$ is embedded in $X=(D, H)$, and if $x \in$ int $X$, then $d\left((\hat{H})^{n}(x),(I, f)\right) \rightarrow 0$. Here $\hat{H}$ is the homeomorphism on $X$ induced by $H$. Furthermore, the homeomorphism $\hat{H} \mid(I, f)$ is just the homeomorphism $\hat{f}:(I, f) \rightarrow(I, f)$, induced by $f$.

Definitions. If $Z$ is a compact metric space, and $g: Z \rightarrow Z$ is continuous, the inverse limit space $(Z, g)$ is $\left\{\left(z_{0}, z_{1}, \ldots\right) \mid z_{i} \in Z\right.$ and $\left.f\left(z_{i+1}\right)=z_{i}\right\}$ with the metric $\rho\left(\left(z_{0}, z_{1}, \ldots\right),\left(y_{0}, y_{1}, \ldots\right)\right)=\sum_{i=0}^{\infty} d\left(z_{i}, y_{i}\right) / 2^{i}$. The in-

Received by the editors March 21, 1989 and, in revised form, November 13, 1989.

1980 Mathematics Subject Classification (1985 Revision). Primary 58F13, 58F22, 55F20, $54 \mathrm{H} 20$. 
duced homeomorphism $\hat{g}:(Z, g) \rightarrow(Z, g)$ is given by $\hat{g}\left(\left(z_{0}, z_{1}, \ldots\right)\right)=$ $\left(g\left(z_{0}\right), z_{0}, z_{1}, \ldots\right)$.

If $A$ is a subset of the plane $E^{2}$, the statement that $A$ is a global attractor means that there is a homeomorphism $h: E^{2} \rightarrow E^{2}$ such that (1) $h(A)=A$; (2) if $x \in E^{2}$ then $d\left(h^{n}(x), A\right) \rightarrow 0$ as $n \rightarrow \infty$ and; (3) if $U$ is open and $A \subset U$, then there is an open set $V$ and a positive integer $N$ such that $A \subset V \subset U$ and if $n>N$, then $h^{n}(V) \subset U$.

Construction of the examples. For $i=1,2,3$, let $B_{i} \subset E^{2}$ be $\{(x, y) \mid-i \leq$ $x \leq i$ and $-i \leq y \leq i\}$. Let $I$ be the interval $\{(t, 0) \mid-1 \leq t \leq 1\}$ and suppose that $f: I \rightarrow I$ is continuous.

Now let $h: B_{3} \rightarrow B_{3}$ be a homeomorphism such that (1) $h \mid B_{3}-B_{2}=$ id; (2) if $(t, 0) \in I$ then $h((t, 0))=(f(t), t)$. This last condition insures that $h$, followed by vertical projection onto $I$, is $f$. See the diagram.

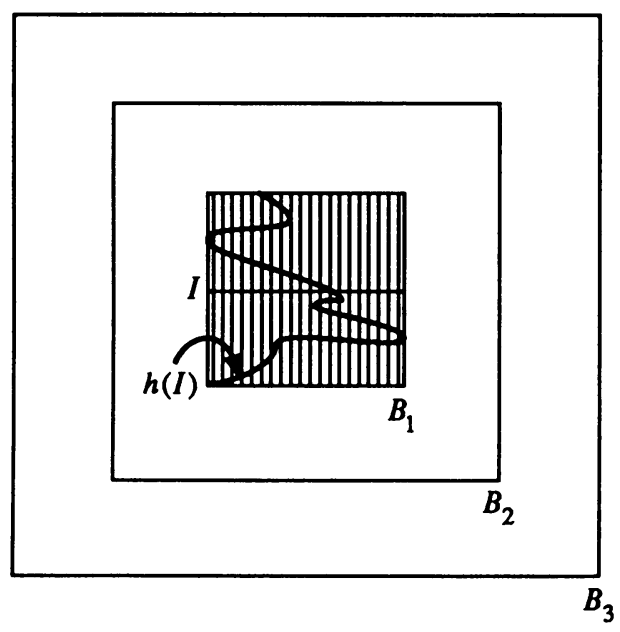

We now construct a continuous function $G: B_{3} \times[0,1] \rightarrow B_{3}$. Denoting $G \mid B_{3} \times\{t\}$ by $G_{t}$, we will have the following properties:

(1) $G_{0}=\mathrm{id}$

(2) $G_{t}$ is a homeomorphism if $0 \leq t<1$;

(3) for each $t, G_{t} \mid \operatorname{Bdry}\left(B_{3}\right)=$ id ;

(4) if $(t, 0) \in I$, then $\{(t, s) \mid-1 \leq s \leq 1\} \subset G_{1}^{-1}((t, 0))$;

(5) $G_{1}\left(B_{2}\right)=B_{1}$;

(6) if $x \in$ int $B_{3}$, there is an integer $n$ such that $G_{1}^{n}(x) \in I$.

Roughly speaking, $B_{2}$ is gradually squeezed down to $B_{1}$ while the vertical 
intervals in $B_{1}$ are shrunk down to points in $I$.

Now, let $H=G_{1} \circ h$ and let $X=\left(B_{3}, H\right)$ be the inverse limit space of $B_{3}$ with bonding map $H$. From Condition (2), it follows that $H$ is uniformly approximated by homeomorphisms. Using [ $\mathrm{Br}$, Theorem 4], it follows that $X$ is a topological disk. Let $A=\left\{\left(x_{0}, x_{1}, \ldots\right) \mid\left(x_{0}, x_{1}, \ldots\right) \in X\right.$ and $\left.x_{i} \in I\right\}$. Then $A \subset X$, and it follows from (5), (6), and the fact that $h=$ id on $B_{3}-B_{2}$, that $A$ is a global attractor for int $X$ under $\hat{H}$.

Now suppose that $(t, 0) \in I$. Then $H(t, 0)=G_{1}((f(t), t))=(f(t), 0)$, by (4). From this it follows that $A$ is homeomorphic with $(I, f)$. Notice that if $\left(x_{0}, x_{1}, \ldots\right) \in A$, then $\hat{H}\left(\left(x_{0}, x_{1}, \ldots\right)\right)=\left(H\left(x_{0}\right), x_{0}, x_{1}, \ldots\right)=$ $\left(f\left(x_{0}\right), x_{0}, \ldots\right)=\hat{f}\left(\left(x_{0}, x_{1}, \ldots\right)\right)$. so the homeomorphism induced on $A$ by $\hat{H}$ is just $\hat{f}$.

Remarks. Notice that if $\rho \in$ int $X$, then there is a point $q \in A$ such that $d\left((\hat{H})^{n}(p),(\hat{H})^{n}(q)\right) \rightarrow 0$. Also it is clear that this construction, and elaborations of it, can be carried out in much greater generality. We will discuss these results elsewhere.

\section{REFERENCES}

[B-M1] M. Barge and J. Martin, Chaos, periodicity, and snakelike continua, Trans. Amer. Math. Soc. 289 (1985), 355-363.

[B-M2] _ Dense orbits on the intervals, Michigan Math. J. 34 (1987), 3-11.

[B-M3] _ Dense periodicity on the interval, Proc. Amer. Math. Soc. 94 (1985), 731-736.

[Bi] R. H. Bing, Snakelike continua, Duke Math. J. 18 (1951), 653-663.

[Br] M. Brown, Some applications of an approximation theorem for inverse limits, Proc. Amer. Math. Soc. 11 (1960), 478-483.

[Wa] W. Watkins, Homeomorphic classification of certain inverse limit spaces with open bonding maps, Pacific J. Math. 103 (1982), 589-601.

[Wi] R. F. Williams, One-dimensional non-wandering sets, Topology 6 (1967), 473-483.

Department of Mathematics, Montana State University, Bozeman, Montana 59717

Department of Mathematics, University of Wyoming, LaRamie, Wyoming 82071 